\title{
Ultrastructural changes in female reproductive organ of Chrotogonus trachypterus Blanchard induced by deltamethrin
}

\author{
Shashi Meena ${ }^{1} \&$ N. P. Singh ${ }^{2}$ \\ Centre for Advanced Studies in Zoology, University of Rajasthan, Jaipur-302055, Rajasthan, India
}

\begin{abstract}
Acridid grasshopper, Chrotogonus trachypterus Blanchard is known as surface grasshopper and is a most common polyphagous pest occurring throughout year causing significant damage to seedlings of crops and vegetables. Ultrastructural changes in the ovarian follicles of C. trachypterus Blanchard induced by deltamethrin one day after treatment were observed. Orthopteran insects have panoistic ovarioles and each of the paired ovary consists of tubular ovarioles along which are placed the oocytes in linear sequence that reflexes their progressive development. Each ovariole is divided into a terminal filament, germarium and a vitellarium. In the present study electron micrographs of ovarian follicle cells of females treated with deltamethrin showed prominent histopathological changes leading to vacuolization of cytoplasm, degeneration of the cell components of follicular epithelium and most obvious signs were observed of yolk damage and mitochondrial disintegration, when examined by transmission electron microscopy (TEM). The present study indicates a profound effect on reproduction of the pest by deltamethrin, a synthetic pyrethroid and suggests alternative of more hazardous synthetic organic insecticides.
\end{abstract}

Key Words: Chrotogonus trachypterus Blanchard, ovarian follicle, synthetic pyrethroid, deltamethrin histopathological changes, transmission electron microscopy (TEM)

\section{Introduction}

The surface grasshopper, Chrotogonus trachypterus Blanchard (Orthoptera: Acrididae) has been recognized as a threat to agricultural in semi arid zone of Rajasthan, India. It is a destructive polyphagous pest of crops in seedling stage. Adults as well as nymphs cause severe damage to cereals and vegetables [1], [2], [3], [4]. Locust and grasshopper control has been carried out with chemical pesticides since ancient time. Although chemical pesticides have brought spectacular revolution in grasshopper control, however, concern about their negative impact on the environment caused them to be prohibited in most countries. Alternative of these, the use of synthetic pyrethroids has rapid knockdown effect on insect pest. Contact toxicity of synthetic pyrethroids alter morphology and cause cellular degeneration in insects. Deltamethrin is one of the synthetic pyrethroids which have extremely high insecticidal activity at very low concentration and biodegradable in nature have been introduced to suppress the development and reproduction of insect pest. Very low application rate of synthetic pyrethroids brings reduced environmental pollution. Exposure of sublethal doses of the insecticide greatly affected the development of gonads in insects [5], [6], [7], [8], [9]. Although, the use of pesticides has proved quite effective in the grasshopper management, but not much work has so far been done for the control of $C$. trachypterus using synthetic pyrethroids. Hence, the present work was aimed to investigate histopathological effects in ovaries of $C$. trachypterus induced by sub lethal concentration $\left(\mathrm{LC}_{50}\right)$ of deltamethrin.

\subsection{Experimental insect}

\section{Materials and Methods}

The stock culture of $C$. trachypterus was maintained in laboratory at $30 \pm 2^{\circ} \mathrm{C}$ temperature and $60 \pm 5 \%$ RH at Entomology Laboratory, Department of Zoology, University of Rajasthan, Jaipur, Rajasthan (India).

\subsection{Insecticides used}

Synthetic pyrethroid- Deltamethrin ( $\left.\mathrm{LC}_{50}=18.36 \mathrm{ppm}\right)$

Adult females were topically treated with sub lethal concentration of deltamethrin using a micro syringe and dissected 24 hour after treatment.

\subsection{Transmission Electron Microscopy (TEM)}

Ovarioles were removed/excised from the abdomen and cleaned from the surrounding fat body, then kept in ice-cold $\left(0-5{ }^{\circ} \mathrm{C}\right)$ karnovsky fixative, $\mathrm{pH} 7.3$ for electron microscopy. The tissues were transferred to

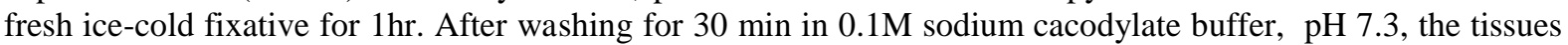
were post-fixed for further $1 \mathrm{~h}$ in $1 \%$ osmium tetroxide in $0.1 \mathrm{M}$ sodium cacodylate buffer at same $\mathrm{pH}$ at $4 .{ }^{\circ} \mathrm{C}$.The samples were then dehydrated at room temperature via a graded series of ethanol solutions to 
propylene oxide prior to embedding in Araldite epoxy resin. Semi thin sections were cut from these blocks stained with toluidine blue and examined under the light microscope. The ultrathin sections so obtained from selected blocks were mounted on copper grids stained with uranyl acetate and lead citrate and then viewed in a transmission electron microscope (Philips Morgagni 268 D) at the central laboratory, Sophisticated Analytical Instrumentation Facility (DST), Department of Anatomy, All India Institute of Medical Sciences (AIIMS), New Delhi.

\begin{abstract}
III. Results
3.1 Structure of ovarian follicle of normal adult

The female reproductive system of $C$. trachypterus consists of a pair of ovary either side in the abdomen, is consisted of a number of egg-tubes or ovarioles which are placed the oocyte in a linear sequence and reflects their progressive development (Fig.1). The elongated tube in which the developing eggs are disposed one behind the other in a single chain, the oldest oocyte being situated near the union with the oviduct. The wall of an ovariole is made of inner tunica propria which rests upon a basement membrane. Outside the latter is a peritoneal coat of connective tissues (Fig. 4). In C. trachypterus panoistic type of ovarioles are found in which nurse cells are absent. As oocyte grows, its nucleus increases proportionately in size and it is known as the germinal vesicle. Each ovariole is consisted of variable number of oogonia and oocytes at different stages of development.
\end{abstract}

\title{
3.2 Histologically three zones are differentiated in an ovariole:-
}

Terminal filament is a slender thread like apical prolongation of the peritoneal layer and unites with each other to form the suspensory ligament which holds the ovary in its position.

The narrow terminal part of each ovariole, the germarium is differentiated in to cells that were destined to become the oocyte. In the germarium, oogonia and the pre vitelline oocytes were found. The oogonia showed various stages of cell division (Fig.2). Pre vitelline oocytes were present at the periphery and were smaller than the oogonia. The oocytes are arranged in linear fashion one behind the other and are surrounded by the distinct follicular epithelium. The size of oocytes increased from terminal end to down wards of the ovariole. Thus, the younger oocytes were found towards the germarium or apical end.

The vitellarium which constitutes the major portion of an ovariole contains the developing oocytes at different stages of development. The epithelial layers of the wall of the vitellarium grows inwards and enclose each oocyte in a definite sac or follicle thus, each ovarian follicle is composed of oocytes with surrounding follicular epithelium (Fig. 4). During vitellogenesis reserve material was deposited in the ooplasm, consequently, the oocyte is filled with numerous yolk spheres. The oocyte nucleus became ovoid and is shifted to a lateral side.

The ultrastructural study revealed that the follicular epithelium or peritoneal sheath of vitellogenic follicle was composed of a single layer of more or less columnar to cuboidal cells containing homogenous yolk material. The matrix contained yolk spheres and clear spaces near the adjusting follicular cells. Follicular cells play a major role in the incorporation of yolk granules into the oocyte during vitellogenesis. Normal follicular cells appeared with homogenous yolk material. Follicle cells exhibited abundance of oval shaped mitochondria, smooth and rough endoplasmic reticulum and golgi bodies in the cytoplasm (Fig. 5, 6). In ultastructural observation normal follicular cells appeared with homogenous ooplasm and mitochondria contained normal cristae. Presence of well developed mitochondria proved normal protein synthesis and secretion of yolk during vitellogenesis. Follicle cell nuclei were large and occupied most of the cell volume throughout vitellogenesis. Coated vesicles were also detected between follicular cells indicating their role in the selective uptake of protein (Fig. 7). During vitellogenesis the nucleus and nuclear membrane appeared normal and cytoplasm was homogenous with rich mitochondria. The nuclei were spherical in shape and bound by well developed nuclear membrane. Chromatin material was evenly distributed in nucleus (Fig. 6). Mature oocytes were characterized by having an abundance of large yolk bodies each of which was surrounded by a granulated rim.

\subsection{Structure of ovarian follicle of treated adult}

Ultrastructural histopathological examination of ovarian follicles one day after treatment with sublethal concentration of deltamethrin showed different deteriorating effects. Vacuolization in developing ovarian follicles exhibited degeneration of cell components with damaged yolk. Yolk material in oocyte was observed no longer homogenous and showed clear separation. Many vacuoles appeared in yolk region indicating abruption of vitellogenesis (Fig. 9). In oocytes, the yolk bodies were surrounded by relatively clear rings, some of which showed cracks and fissures dividing the yolk bodies, while others appeared normal. Large lipid droplets of different sizes were also present in the oocytes (Fig. 9). There was disorganization of mitochondria with malformation of their structure without cristae (Fig. 10). The ooplasm was no longer homogenous, the nucleus appeared with many lysosome- like bodies and phagocytic vacuoles indicating the beginning of cell 
lysis (Fig. 10). Finally, it was revealed that electron micrograph of ovarioles showed degeneration of cell components of the follicular epithelial cytoplasm, aggregation of mitochondria and the vacuolized ooplasm.

\section{Discussion}

The ovarioles found in Chrotogonus trachypterus Blanchard panoistic type, in which nurse cells are absent and ovarioles are differentiated into three zones viz., the terminal filament, germarium and vitellarium. Engelmann [10] and Reda [11] reported that Schistocerca gregaria as well as other orthopteran insects have panoistic ovarioles enclosed in a nucleated ovarian sheath. Similarly, Tobe and Pratt [12] observed four distinct periods, an early growth period, previtellogenic period, vitellogenic period and finally chorionation, which preceeds ovulation in Schistocerca gregaria. Simiczyjew and Margas [13] also revealed ovaries of Ischnopsyllus spp. was composed of panoistic ovarioles comprised of a terminal filament, germarium, vitellarium and pedicel. During vitellogenesis reserve materials were deposited in ooplasm and consequently oocytes become voluminous and filled with numerous yolk spheres. Ghazawi et al [7] also reported that female of Heteracris littoralis Ramb. consists of similar ovarian structure.

Electronmicrograph revealed homogenous matrix containing yolk spheres and clear spaces near the adjoining follicular cells. Follicle cells exhibited abundance of mitochondria, endoplasmic reticulum and golgi bodies in the cytoplasm. Coated vesicles were also detected between follicular cells which proved to be engaged in the selective uptake of protein in the ovarian follicles of C. trachypterus. John and James [14] observed that the apical ooplasm of S. gregaria was rich in ribosomes and mitochondria, while rough endoplasmic reticulum (rER) and golgi complexes occupied the basal and basolateral regions of the cells. These ultra structures point to an increase in the activity of follicular cells associated with the synthesis and secretion of egg precursors. Bilinski and Büning [15] reported panoistic oocyte nuclei (germinal vesicles) of previtellogenic oocytes contained numerous polymorphic multiple nucleoli, chromatin aggregations and within the latter phase, abundant ribosomes as well as mitochondria in the snow scorpionfly, Boreus hyemalis. Dos Santos and Gregorio [16] reported a population of lumen cells, with morphological features of secretory activity between the epithelial sheath and the tunica propria.

Smith [17] revealed that electron microscope can play role in clarify and bringing into sharper focus aspect of coated vesicles on the basis of their known biochemical and physiological attributes. They proved their engagement in the selective uptake of particular protein. Anderson [18] described protein yolk formation in the cockroach, Periplaneta americana as regard the role of coated vesicle in build up yolk granules within the ooplasm. The junctional complex presumably help to maintain intracellular communication and the structural integrantion of the epithelium, but the fate of junctional complex at the end of yolk deposition vary due to deposition of harmful chemical substances [14] \& [16]. Hussein et al [20] also observed junctional complexes between follicles in S. gregaria and Follicle cells play an important role in the synthesis of yolk components. Among these are glycoproteins, phospholipids and triglycerides [21], using cytochemical techniques Hinton [22] described the occurrence of fatty and compound yolk in oocytes of Aspongopus obscures which is comprised of unsaturated neutral fats synthesized in oocytes. Activity of follicle cells increase the secretion and synthesis of egg envelop precursors as proved by the presence of dictyosomes, rough endoplasmic reticulum and free polyribosomes in Ischnopsyllus spp. [23], [24]. Further, Grandi [25] reported lysosome like bodies, lipid droplets as well as polyribosomes, numerous dense granules, free or associated mitochondrial clusters in ovary of Kalotermes flavicollis (Fabr.) and at meiotic stage the cisternal complex became vacuolated, as evidence of disintegration and during late vitellogenesis the germinal vesicle contains numerous uniformly distributed, tiny multiple nuclei which proved rapid RNA synthesis for formation of yolk.

Ultrastructural histopathological examination showed different deteriorating effects on oocytes. Follicular cells were degenerated and vacuolization was exhibited in developing ovarian follicles. Degeneration of yolk region indicated abruption of vitellogenesis. Disorganization of mitochondria with malformation of their structure without cristae was also exhibited. Similarly, newly emerged 5th instar nymphs of S. gregaria treated with cascade, rice bran extract and karate, synthetic pyrethroids each at $\mathrm{LC}_{50}$ produced disturbance in protein synthesis of the ovary, which reflected an inhibition of ovarian maturation and showed a degeneration of ovarioles and oocytes, disintegrated mitochondria, enlarged vacuoles and cracked yolk bodies mostly in two halves [14], [19] \& [20]. Ghazawi et al [7] observed disintegration and destruction of ovarian follicle cells and mitochondria in topically treated female of Heteracris littoralis with serial concentrations of azadirachtin. According to Reda [11] many histopathological changes in ovaries of S. gregaria produced disturbance in protein synthesis which reflected an inhibition of ovarian maturation and degeneration and vacuolization of the follicular cells when treated with chitin inhibitor, consult and lufox toxicants. Vitellogenesis was inhibited in mature oocytes. The follicular epithelial cells were greatly malformed and lysis of cytoplasm was observed.

According to Gill et al [26] synthetic pyrethroids led to degeneration and toxic changes in reproductive organs. Cypermethrin treatment induced tissues alterations which included degenerative changes in luteal cells, pleomorphic changes, nuclear degeneration and vacuolation, cell shrinkage and rupture, cloudy swelling and 
hydropic degeneration, less cytoplasmic granulation, cell elongation, hyalinization and cytoplasmic haziness and stripling and necrosis. Synthetic pyrethroids have a profound effect on the central nerve cord by plugging of sodium and potassium channels, which ultimately stimulate the endocrine system and later on it may affect development of reproductive system [27] \& [28]. Follicular cells of developing oocyte apparently regulate transfer of materials for oocyte growth as histochemical studies have proved in the telotrophic ovarioles of Crynodes peregrinus. Follicle cells synthesize RNA which is exported to oocytes during its early stages of growth [29].

During yolk deposition, as the oocyte grows much more rapidly, the germinal vesicle becomes relatively smaller and finally, the nuclear membrane breaks down. Peaks of synthetic activity in the corpus allatum correspond well with the onset of previtellogenic growth in the oocytes. Corpus allatum insufficiency is responsible for resorption of growing oocytes [12]. Improper functioning of endocrine system in female insects could be another reason during the maturation of the ovaries. However, according to Ergen [30] the accessory glands required corpora allata for their normal secretory activity. Therefore, the probability of endocrine system affecting the gonads cannot be ruled out.

\section{Conclusion}

The present study revealed that deltamethrin induced toxic effects pertaining to morphological and histopathological changes in female reproductive organ. Most of the histopathological disruptions in gonads were directly related to the fertility and mortality. Thus synthetic pyrethroids could be used as control tool in integrated pest management programme to reduce reproduction of this pest in agricultural area.

\section{Acknowledgements}

The authors wish to acknowledge thankfulness to the Head, Centre for Advanced Studies in Zoology, University of Rajasthan, Jaipur for providing laboratory facilities to carry out the work. University Grants Commission is also thankfully acknowledged for providing financial assistance in the form of research fellowship to Shashi Meena.

\section{References}

[1]. G. S. Grewal and A. S. Atwal, Development of Chrotogonus trachypterus (Blanchard), (Orthoptera, Pyrgomorphidae) in relation to different levels of temperature and humidity, Indian Journal of Entomology, 30(1), 1968, 1-7.

[2]. A. Latif, Pests of cotton and their control, FAO Project Report Training Courses Progress Report, 4, 1975, 345-361.

[3]. B. K. Rai, Pests of oilseed crops in India and their control, Indian Council Agri. Res., New Delhi, 1976, 121 pp.

[4]. A. Singh and K. M. Singh, Succession of insect-pests in early varieties of red gram, Cajanus cajan (L.) Millsp., Indian Journal of Entomology, 40, 1978, 1-6.

[5]. M. D. Gracera, P. Ibanez, R. Martinez and P. Cunat, Effects of juvenile hormone and prococene II on the metabolic rate of the digestive system, fat body and ovaries of the insect Oncopeltus fasciatus. Rev. Espan. Fisiol., 45(4), 1989, 357-362.

[6]. A. Janak, Necrosis induced by an organochlorine pesticide (endosulfan) in the gonads of orthopteran insect, Poecilocerus pictus (Fabr). Proc. Acad. Environ. Bio., 1, 1992, 159-165.

[7]. A. N. Ghazawi, E. D. El-Shranoubi, M. M. El-Shazly and A. Abdel Rahman , Effects of Azadirachtin on mortality and reproductive system of the grasshopper, Heteracris littoralis Ramb. (Orthoptera : Acrididae). Journal of Orthoptera Research, 16(1), 2007, 57-65.

[8]. A. Habluetzel, F. Carnevali, L. Lucantoni, L. Grana, A. R. Attili, F. Archilei, M. Antonimi, A. Valbonesi, V. Abbadessa, F. Esposito and E S. A. Vander, Impact of the botanical insecticide Neem, Azadirachtin on survival and reproduction of the biting louse, Damalinia limbata on angora goats. Vet. Parasitol, 144, 2007, 328-337.

[9]. N. S. Senthil, C. M. Young, S. H. Yul, P. C. Hoon, K. Kalaivani and K. J. Duk, Effect of azadirachtin on acetylcholinesterase (AchE) activity and histology of the brown planthopper, Nilaparvata lugens (Stal.). Ecotoxicol. Environ. Saf., 70, 2008, 244-50.

[10]. F. Engelmann, The physiology of insect reproduction (Pergamon Press, Oxford, 1970).

[11]. F. A. B. Reda, I. M. Mona, M. El-G. Abd Elazeem and M. M. Noura, Histopathological change in the testis of the desert locust, Schistocerca gregaria (Forskal) induced by the IGR Consult and Lufox. Egypt, Acad. Journal of Biological Sciences, 1(1), 2010, 2328.

[12]. S. S. Tobe and G. E. Pratt, Corpus allatum activity in vitro during ovarian maturation in the desert locust, Schistocerca gregaria. Journal of Experimental Biology, 62, 1975, 611-627.

[13]. B. Simiczyjew and W. Margas, Ovary structure in the bat flea, Ischnopsyllus spp. (Siphonaptera: Ischnopsyllidae), Phylogenetic Implications, Zoolog. Polon., 46(1-4), 2001, 5-14.

[14]. C. D. John and T. B. James, Ovarian follicle development during vitellogenesis in the house cricket, Acheta domesticus. Journal of Morphology, 200, 1989, 185-198.

[15]. S. M. Bilinski and J. Büning, Structure of ovaries and oogenesis in the snow scorpionfly, Boreus hyemalis (Linne) (Mecoptera : Boreidae). International Journal of Insect Morphology and Embryology, 27(4), 1998, 333-340.

[16]. D. C. Dos Santos and E. A. Gregorio, Ultrastructure of the ovariole sheath in Diatraea saccharalis (Lepidoptera: Pyralidae), Biocell, 26(2), 2002, 229-235.

[17]. D. Smith, Insect cells. Their structure and function (Oliver and Boyd Ltd. Edinburgh, 1968) pp 100-113.

[18]. E. Anderson, Oocyte differentiation and vitellogenesis in the cockroach, Periplaneta americana. Journal of Cell Biology, 20, 1964, 131-135.

[19]. H. J. Ferenz, Yolk protein accumulation in Locusta migratoria oocytes. Journal of Insect Morphology and Embrology, 22(2-4), 1993, 295-314.

[20]. M .A. Hussein, L. S. Hamouda, R. F.A. Bakr, H. A. Hassan and Z. F. Elsokary, Effect of Cascade, Oryza sativa bran extract and Karate on fine structure of the ovary of Schistocerca gregaria. Egypt. Acad. J. Biol. Sci. 1(1), 2008, $13-21$.

[21]. W. B. Wigglesworth, The principles of insect physiology (Methuen and Co. Ltd. NewYork, NY: Wiley, 1972), 827pp. 
[22]. H.E. Hinton, Accessory function of seminal fluid. Journal of Medical Entomology, 11, 1974, 19-25.

[23]. M. S. Kaulenas, Structure and function of the female accessory reproductive systems (Springer Verlag, Berlin, 1992), 38-82pp.

[24]. J. Buning, The insect ovary. Ultrastucrure, previtellogenic growth and evolution (Chapman and Hall, London, 1994), 40-61pp.

[25]. G. Grandi, Ultrastructural study of testis development and spermatogenesis in Kalotermes flavicollis (Fabr.) (Isoptera : Kalotermitidae). Journal of Morphology, 94(5), 1992, 717-725.

[26]. S. A. Gill, F. Rizvi, M. Z. Khan and A. Khan, Toxic effects of cypermethrin and methamidophos on bovine corpus luteal cells and progesterone production. Department of Veterinary Pathology, University of Agriculture, Faisalabad-38040, Pakistan, 2009.

[27]. H. C. L. Gupta, R. Singh, R. L. Jain and R. C. Saxena, Relative and residual toxicity of some synthetic pyrethroids, Indian Journal of Entomology, 54(1), 1992. 34-38.

[28]. H. C. L. Gupta, Insecticides: Toxicology and uses (Agrotech publishing academy: Udaipur 1999), 92-99 pp.

[29]. A. Ray and P. S. Ramamurthy, Source of RNA supply to oocytes in Crynodes peregrines Fuessly (Coleoptera, Chrysomedidae). International Journal of Insect Morphology and Embryology, 8(2), 1979, 113-122.

[30]. G. R. Ergen, The effects of precocene II on the fine structure of corpus allatum in adult female Anacridium aegyptium L. (Orthoptera, Acrididae), Turk. J. Zool., 25, 2001, 95-103.

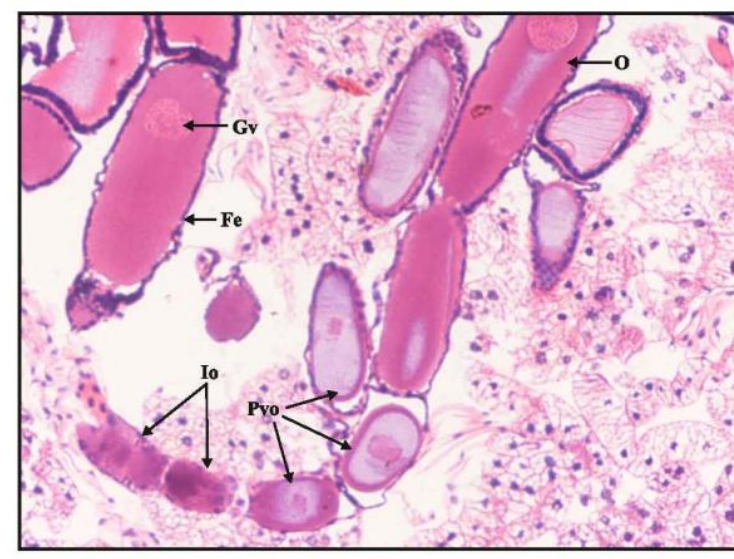

Fig. 1 Light micrograph of normal ovariole of C. trachypterus showing different stages of immature oocyte(Io), pre vitelline oocyte (Pvo), follicular epithelium $(\mathrm{Fe})$, mature oocyte $(\mathrm{O})$, germinal vesicle $(\mathrm{Gv})$. HaematoxylinEosin, 100X

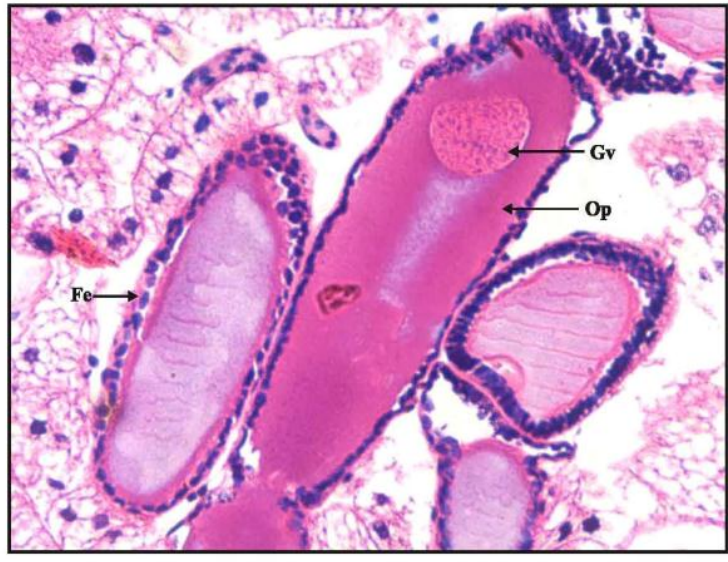

Fig. 3 Higher magnification of normal ovariole of C. trachypterus showin mature oocyte with germinal vesicle $(\mathrm{Gv})$, ooplasm $(\mathrm{Op})$ and follicular epithelium(Fe). Haematoxylin-Eosin, 200X

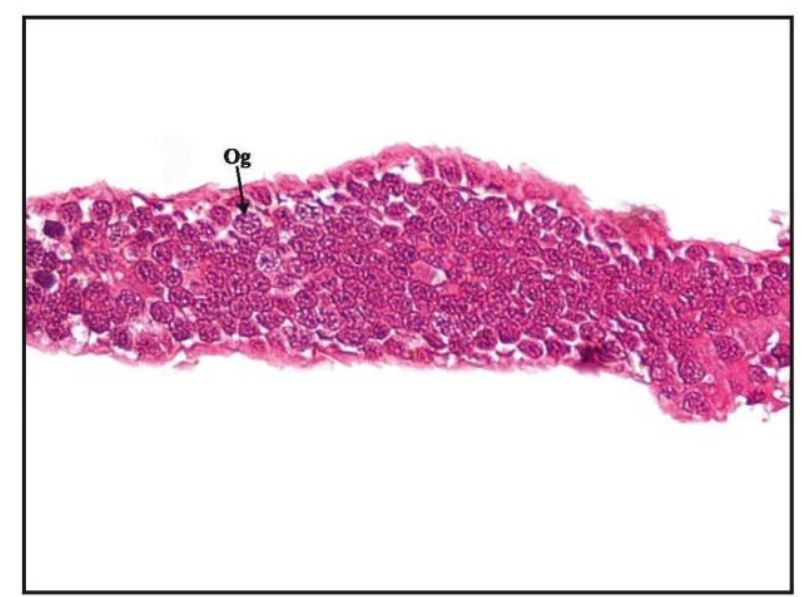

Fig. 2 Light micrograph of normal ovariole of C. trachypterus showing germarium with oogonia (Og). Haematoxylin-Eosin, 200X

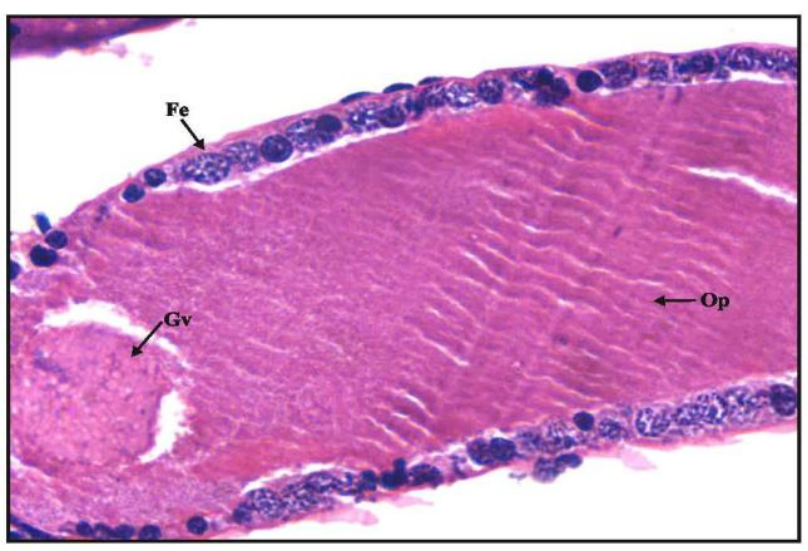

Fig. 4 Light micrograph of normal ovariole of C. trachypterus showing single layer of follicular epithelium $(\mathrm{Fe})$, germinal vesicle $(\mathrm{Gv})$ and ooplasm (Op). Haematoxylin-Eosin, 400X 


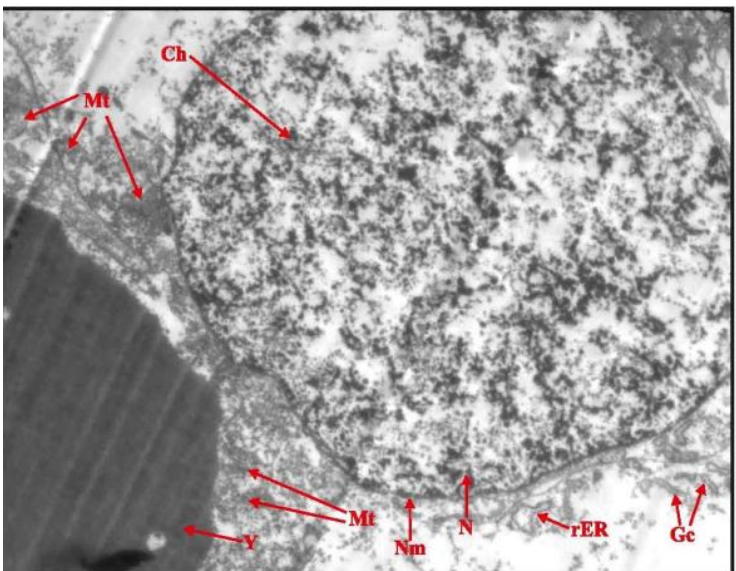

Fig. 5 Electron micrograph of normal ovariole of $C$. trachypterus showing nucleus $(N)$ nuclear membrane (Nm), rough endoplasmic reticulum (rER), mitochondria (Mt), golgi complex (Gc) and chromatin (Ch) material. X1600,

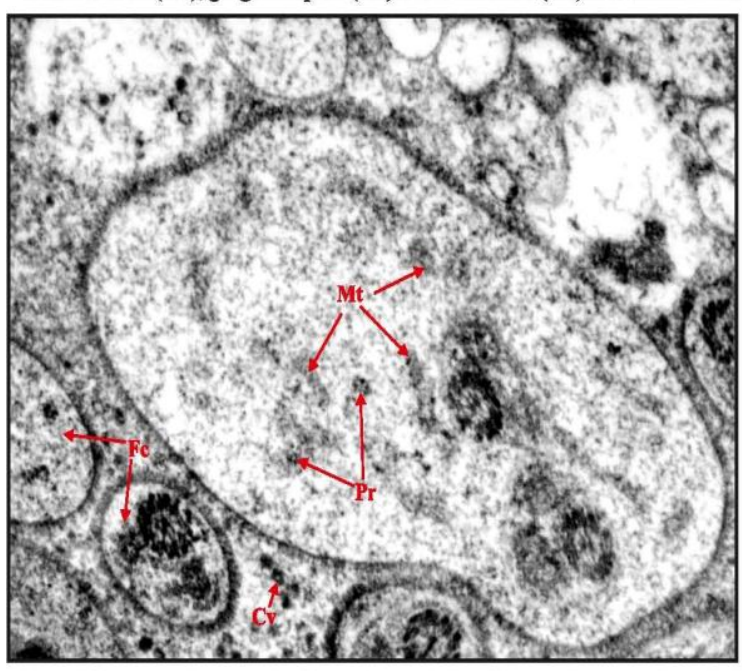

Fig. 7 Electron micrograph of normal ovariole of $C$. trachypterus showing coated vesicles $(\mathrm{Cv})$ in ooplasm and developing ovarian follicular cells $(\mathrm{Fc})$. $\mathrm{X} 8000$

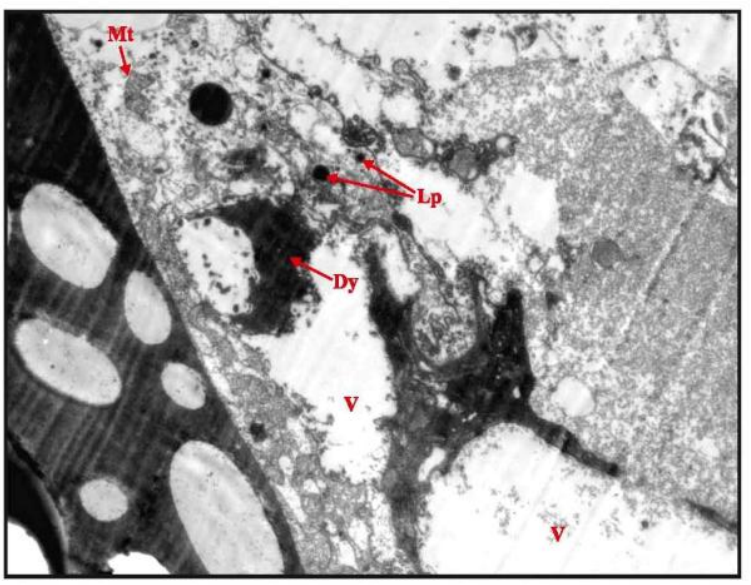

Fig. 9 Electron micrograph of one day treated ovariole of C. trachypterus near the end of vitellogenesis in ooplasm with damaged mitochondria (Mt) without cristae, damaged yolk bodies (Dy), lipid droplets (Lp) and vacuolization(V).X1600

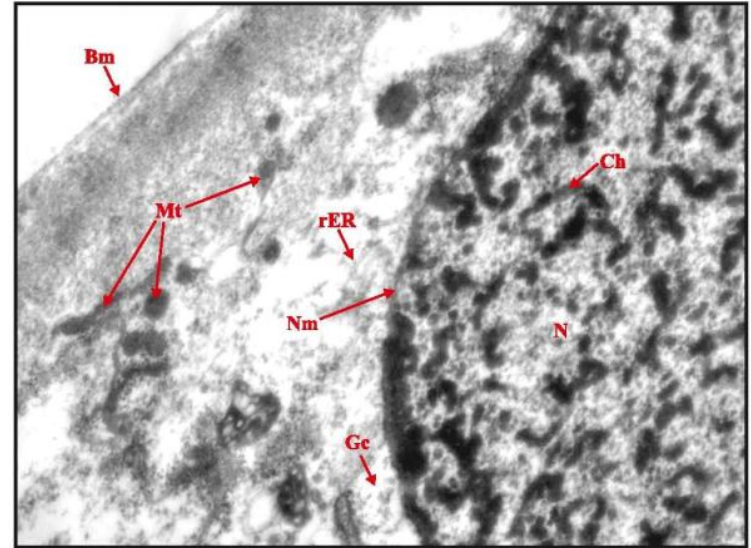

Fig. 6 Higher magnification of normal ovariole of $C$. trachypterus showing nucleus $(\mathrm{N})$, nuclear membrane $(\mathrm{Nm})$, rough endoplasmic reticulum (rER) mitochondria $(\mathrm{Mt})$, golgi complex $(\mathrm{Gc})$, chromatin $(\mathrm{Ch})$ material and basement membrane (Bm). X5000

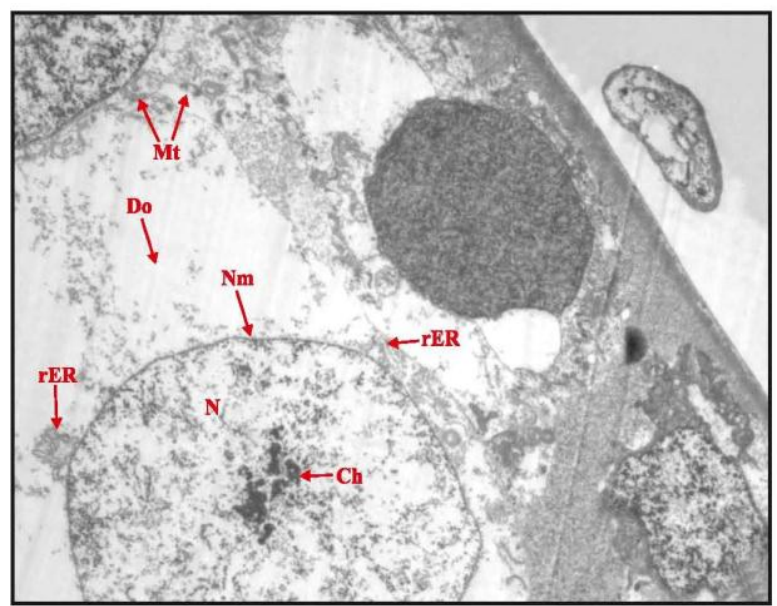

Fig. 8 Electron micrograph of one day treated ovariole of $C$. trachypterus showing nucleus $(\mathrm{N})$, nuclear membrane $(\mathrm{Nm})$, rough endoplasmic reticulum (rER), mitochondria (Mt), condensed chromatin ( $\mathrm{Ch})$ material and degenerated ooplasm(Do). X1000

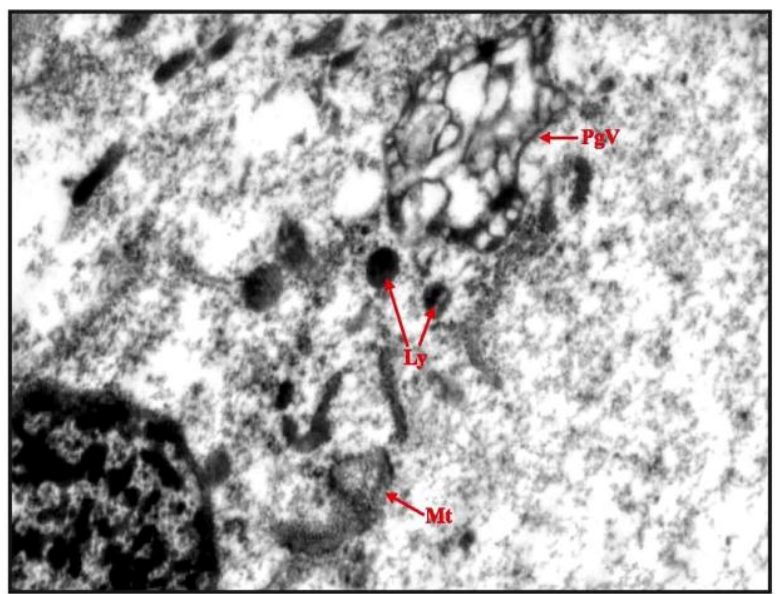

Fig. 10 Electron micrograph of one day treated ovariole of C. trachypterus showing cytoplasm with aggregating mitochondria (Mt) without cristae, lysosomes (Ly) and phagocytic vacuoles (PgV). X1600 